\title{
Rapid mold-free fabrication of long functional PDMS fibers
}

\author{
Jeong Hui Lee', Taekyung Lim (1)', Keumyoung Seo', Jeeyin Park', Jonguk Yang', Sang-Mi Jeong (D) and \\ Sanghyun Ju (1D ${ }^{1}$
}

\begin{abstract}
Polydimethylsiloxane (PDMS), an optically transparent and inert material, is widely used in biological and semiconductor applications owing to its excellent chemical stability and moldability. This study proposes a thermally induced wet spinning method for the fabrication of long PDMS fibers with a constant width. PDMS is a thermoset polymer that undergoes chemical crosslinking when heated, and the thermally induced wet spinning process allows for the formation of fibers without a mold. A rapid thermal curing step was used to instantly solidify the thermoset polymer, where immediate chemical crosslinking of fluid PDMS solution was achieved upon contact with an oil coagulation bath at $180-230^{\circ} \mathrm{C}$. A rapid stretching process was applied to pull out and control the width of the fiber, and the PDMS was stretched at a rate of $1.2-12.5 \mathrm{~m} / \mathrm{min}$ during the crosslinking process. The fabricated pristine PDMS fibers were transparent and maintained a crosslinked network with excellent mechanical strength. In addition, the PDMS fibers were functionalized with silica nanoparticles, carbon nanotubes, and pores to adjust their transparency/ opacity, conductivity, and heat insulation properties, respectively, for various applications. The proposed thermally induced wet spinning method shows promise for overcoming the limitations of existing molding methods, in which the PDMS fibers cannot be lengthened. Furthermore, the process is environmentally friendly and economical owing to the use of edible canola oil, which reduces the volume of harmful solvents and additives during fiber production.
\end{abstract}

\section{Introduction}

Polydimethylsiloxane (PDMS) is a popular thermoset polymer that is typically used as a liquid-phase prepolymer to form an infusible and insoluble polymer network via thermally activated chemical crosslinking. PDMS offers high transparency, biocompatibility, excellent chemical resistance, and formability and is commonly used as a transparent and flexible substrate, soft lithography mold, and microfluidic channel in flexible displays, biomedical products, and microfluidic devices ${ }^{1-5}$. Solution-based PDMS is typically molded using a solid mold, as thermal energy $\left(25-200^{\circ} \mathrm{C}\right)$ must be applied to activate the Ptbased catalyst, and a crosslinking time of $10 \mathrm{~min}$ or more is required to facilitate the chemical reaction between the

\footnotetext{
Correspondence: Sang-Mi Jeong (jeongsm@kgu.ac.kr) or

Sanghyun Ju (shju@kgu.ac.kr)

'Department of Nanoengineering, Kyonggi University, Suwon, Gyeonggi-Do 16227, South Korea

These authors contributed equally: Jeong Hui Lee, Taekyung Lim
}

polymer chains ${ }^{6,7}$. This method can be used to easily manufacture objects of various sizes and complex shapes; however, the production of a thin and long shape, such as fiber, remains a challenge. One method for producing fibers involves injecting the PDMS precursor into a tube mold and removing the solidified PDMS from the tube mold after crosslinking ${ }^{8-10}$. However, the use of a tube mold limits the diameter of the PDMS fiber and does not allow for lengthening, as the shape of the fiber is determined by the length and inner diameter of the tube mold.

Alternatively, wet spinning and electrospinning methods, which are used to fabricate polymer-based fibers, can be considered to fabricate PDMS fibers. However, the nozzle can become clogged during the spinning process if the PDMS solution undergoes crosslinking too quickly; however, the PDMS fiber can be insufficiently solidified if crosslinking occurs too slowly. Furthermore, because the normal process temperature of wet spinning is $60^{\circ} \mathrm{C}$ or less, PDMS cannot be sufficiently solidified via a chemical 
crosslinking reaction. To solve this problem, a PDMS fiber with a core-shell structure has been fabricated, in which an easily solidified thermoplastic polymer is formed as an outer layer and uncured fluid PDMS is used as an inner layer using the electrospinning method ${ }^{8,11}$. In this case, after postcrosslinking the inner uncured PDMS, only the outer thermoplastic polymer is dissolved and removed to prepare the PDMS fibers. However, this method produces opaque PDMS fibers, and the fibrous morphology and electrospinnability of PDMS are dependent on changes in the PDMS solution over time after preparation; thus, it is difficult to produce fibers with a uniform width.

This study aimed to develop a simple and rapid method for the fabrication of transparent and long PDMS fibers based on thermally induced wet spinning, which is a modification of the conventional wet spinning method. The thermally induced wet spinning method included a hightemperature thermal curing process using a coagulation bath with oil at temperatures of $180-230^{\circ} \mathrm{C}$, while a rapid stretching process was conducted at a speed of $1.2-12.5 \mathrm{~m} /$ min during spinning. A bio-oil with a high boiling point was used to rapidly cure PDMS to form transparent fibers; this resolved the issues associated with the generation of solvent vapor during fiber manufacturing using the conventional wet spinning method. The fiber width was controlled by adjusting the pulling speed of the cured PDMS fiber during rapid stretching. Silica nanoparticles (NPs), carbon nanotubes (CNTs), and poly(methyl methacrylate) (PMMA) particles were embedded in transparent and flexible pristine PDMS fibers to achieve properties, such as transparency/ opacity, conductivity, and thermal insulation.

\section{Materials and methods}

Thermally induced wet spinning of the pristine PDMS fiber

The PDMS solution was extruded from a syringe into a high-temperature coagulation bath and immediately cured to form pristine PDMS fibers. The PDMS solution was prepared by mixing PDMS prepolymer and a curing agent (Sylgard 184, Dow Chemical Company, USA) at a mass ratio of 10:1. The solution was placed in a vacuum oven (SHVDO-08NG, SH Scientific, South Korea) at a negative pressure of 600 Torr for $5 \mathrm{~min}$ to remove air bubbles. The PDMS solution was transferred to a $20-\mathrm{mL}$ syringe, which was mounted on a syringe pump (NE-1000, New Era Pump Systems Inc., USA). To prevent clogging due to the solidification of the PDMS in the needle $(20 \mathrm{G})$, the syringe tip was placed $20 \mathrm{~mm}$ above the surface of the heated canola oil $\left(180-230^{\circ} \mathrm{C}\right)$ in the coagulation bath. As the oil to be used for curing PDMS resin, canola oil, which is not miscible with PDMS resin, has a high smoke point of $240-250^{\circ} \mathrm{C}$ and generates the least toxic gas during the process. The PDMS solution was extruded at a rate of $60 \mathrm{~mL} / \mathrm{h}$, and the linear speed was adjusted between 1.2 and $12.5 \mathrm{~m} / \mathrm{min}$. The cured PDMS fiber was sonicated in a cleaning bath containing isopropyl alcohol (IPA; Daejung, South Korea) for $30 \mathrm{~min}$ to remove all remaining oil from the fiber surface. Although IPA was used to increase the efficiency of fabrication through quick cleaning and drying, water-diluted rubbing alcohol or liquid hand soap could also be used instead of IPA to clean the oil remaining on the PDMS fiber.

\section{Fabrication of the silica-NP-embedded PDMS fiber}

The surface of the PDMS fiber was embedded with a layer of silica NPs to achieve the ability to reversibly switch between being transparent and opaque by adjusting the ratio of mechanical tension. PDMS fibers were fabricated using the thermally induced wet spinning method with a $20 \mathrm{G}$ needle, an oil bath temperature of $230{ }^{\circ} \mathrm{C}$, a syringe pump rate of $60 \mathrm{~mL} / \mathrm{h}$, and a linear speed of $2.5 \mathrm{~m} / \mathrm{min}$. Monodispersed nonporous silica NPs (diameter $=400 \mathrm{~nm}$; Sigma-Aldrich, USA) were mixed in ethanol (Daejung) at a concentration of $10 \mathrm{wt} \%$ via sonication for $30 \mathrm{~min}$. The silica NP solution $(1 \mathrm{~mL})$ was transferred to an airbrush (DH-102, Sparmax, Taiwan) with a $0.25 \mathrm{~mm}$ nozzle and sprayed onto the PDMS fiber at a distance of $100 \mathrm{~mm}$ and a pressure of $0.12 \mathrm{MPa}$. The silica NP-spray-coated PDMS fiber was dipped in PDMS solution and dried in an oven at $60^{\circ} \mathrm{C}$ for $3 \mathrm{~h}$.

\section{Fabrication of the PDMS-CNT composite fiber}

A PDMS-CNT composite fiber was prepared to achieve electroconductivity. Briefly, $1 \mathrm{wt} \%$ multiwalled CNTs (MWCNTs; CNT MR99, Carbon Nanomaterial Technology Co., Ltd, South Korea) were added to benzene (melting point $=5.5^{\circ} \mathrm{C}$; Sigma-Aldrich) and sonicated for 30 min to evenly disperse the CNTs. The PDMS prepolymer was added to the CNT solution and sonicated for 30 min to prepare PDMS-CNT solutions with CNT solution: PDMS prepolymer mass ratios of 101:100, 127:100, 152:100, and 178:100. The PDMS-CNT solution was frozen at $-85^{\circ} \mathrm{C}$ for $8 \mathrm{~h}$ in a freezer (DF8502S, Ilshin Biobase, South Korea) and dried at $-85^{\circ} \mathrm{C}$ and 300 Torr for $15 \mathrm{~h}$ using a freeze dryer (TFD8503, Ilshin Biobase) to remove the remaining benzene. The curing agent was added at $10 \mathrm{wt} \%$ of the prepolymer, and the final concentrations of the CNTs in the PDMS-CNT composite solution were $1.0,1.25,1.5$, and $1.75 \mathrm{wt} \%$. Notably, a PDMS-CNT composite solution with a CNT content higher than $1.75 \mathrm{wt} \%$ was too viscous for the fabrication of fibers. PDMS-CNT composite fibers were fabricated using the thermally induced wet spinning method with a $20 \mathrm{G}$ needle, oil bath temperature of $230^{\circ} \mathrm{C}$, syringe pump rate of $60 \mathrm{~mL} / \mathrm{h}$, and linear speed of $2.5 \mathrm{~m} / \mathrm{min}$.

\section{Fabrication of the porous PDMS fiber (PPF)}

A porous PDMS fiber was formed by adding heteropolymer particles, namely, PMMA, to the PDMS solution. The fiber was formed, and the heteropolymer was 
dissolved from the fiber to leave a microsized open skeleton structure within the PDMS fiber. PMMA $(<15,000$ molecular weight; Sigma-Aldrich) was ground to a powder (particle size: $30-70 \mu \mathrm{m}$ ) using an agate mortar and pestle. The PMMA powder was added to the PDMS solution at concentrations of 10 to $50 \mathrm{wt} \%$ to produce a PDMS-PMMA solution, and air bubbles were removed at a negative pressure of 600 Torr for $5 \mathrm{~min}$. PDMS-PMMA fibers were fabricated using the PDMS-PMMA solution via the thermally induced wet spinning method with a $17 \mathrm{G}$ needle, an oil bath temperature of $230^{\circ} \mathrm{C}$, a syringe pump rate of $60 \mathrm{~mL} / \mathrm{h}$, and a linear speed of $2.5 \mathrm{~m} / \mathrm{min}$. PMMA was removed from the PDMS-PMMA fiber via sonication in IPA for $1 \mathrm{~h}$. The dissolution of the PMMA particles in the PDMS-PMMA fiber left a microsized open structure inside the fiber. The porous PDMS fiber was dried at room temperature $\left(20^{\circ} \mathrm{C}\right)$ for $3 \mathrm{~h}$.

\section{Characterization of the four types of PDMS fibers}

The diameter, cross-section, surface structure, and internal structure of the fabricated pristine PDMS fiber, silica-NP-embedded PDMS fiber, PDMS-CNT composite fiber, and porous PDMS fiber were observed using fieldemission scanning electron microscopy (FE-SEM; S-4800, Hitachi, Japan). The thermal properties of the fibers were determined using thermogravimetric analysis (TGA; TGA/DSC 1, Mettler Toledo, USA) within a temperature range of $20-1000^{\circ} \mathrm{C}$. The change in stress of the fibers according to applied strain was determined using a universal testing machine (UTM; TD-U01, T\&DORF Inc., South Korea). The electrical conductivity of the fibers (length: $10 \mathrm{~mm}$ ) was measured using a digital multimeter (FLUKE-175 EJKCT, Fluke, USA). The strain-sensing properties during repeated stretching from 20 to $80 \%$ were measured using a semiconductor device parameter analyzer (B1500A, Agilent, USA). The internal porosity of the fiber was measured using a porosity meter (AutoPore V 9600, Micromeritics, USA). The thermal insulation properties of the porous PDMS fibers with four different pore numbers were evaluated by fabricating and analyzing six fabrics, namely, PPF-1, 2, 3, and 4 (one layer); 4 (two layers); and 4 (three layers); the dimensions were $11 \times$ $30 \mathrm{~mm}^{2}$. Fabrics woven with porous PDMS fibers (PMMA: 10, 20, 30, and $40 \mathrm{wt} \%$ ) with four different pore numbers were designated PPF-1, 2, 3, and 4. In addition, the fabrics woven with porous PDMS fibers produced with 40\% PMMA were laminated in two and three layers and were named PPF-5 and 6, respectively. The samples were mounted on a plastic frame (thickness: $\sim 1.8 \mathrm{~mm}$ ) with a central open area $\left(75.5 \times 25.5 \mathrm{~mm}^{2}\right)$. The lower part of the sample was exposed to various temperatures from -15 to $100^{\circ} \mathrm{C}$, and the surface temperature of the upper part of the sample was measured using an infrared (IR) camera (T-530, FLIR Systems, Inc., USA). A hot plate
(MSH-20D, DAIHAN Scientific, South Korea) was used as the heating source for temperatures between 40 and $100^{\circ} \mathrm{C}$, and a Peltier module was used as the cooling source for $-15{ }^{\circ} \mathrm{C}$. The thermal conductivity was measured by using a transient plane heat source thermal conductivity meter (TPHS-1, YEONJIN S-TECH, South Korea). The measurement was repeated three times to extract the mean and standard deviation.

\section{Color analysis of the silica-NP-embedded PDMS fiber}

The color change achieved during stretching of the silica-NP-embedded PDMS fiber was evaluated based on the red, green, and blue (RGB) value and the International Commission of Illumination (CIE) $L^{*} a^{*} b^{*}$ color space method. The fiber was imaged under fixed lighting conditions at various degrees of stretching. The images were analyzed using Adobe Photoshop CS4 to extract the RGB and CIE $L^{*} a^{*} b^{*}$ color space values of the samples ${ }^{12}$. The RGB value indicates the intensities of red, green, and blue in the color space. The CIE $L^{*} a^{*} b^{*}$ value includes an $L^{*}$ parameter for the lightness of the sample, ranging from 0 (black) to 100 (white), an $a^{*}$ parameter for color between red $(+)$ and green $(-)$, and a $b^{*}$ parameter for the color between yellow $(+)$ and blue $(-)$. The total color difference $(\Delta E)$ was calculated based on a standard sample $\left(L_{0}\right.$, $\left.a_{0}, b_{0}\right)$ in the neutral state before stretching ${ }^{12,13}$ :

$$
\Delta E=\left[\left(\Delta L^{*}\right)^{2}+\left(\Delta a^{*}\right)^{2}+\left(\Delta b^{*}\right)^{2}\right]^{1 / 2}
$$

where $\Delta L^{*}=L-L_{0}, \Delta a^{*}=a-a_{0}$, and $\Delta b^{*}=b-b_{0}$.

\section{Results and discussion}

Figure 1a shows a schematic of PDMS fiber fabrication using PDMS solution without a mold by the developed thermally induced wet spinning method. To make PDMS act as a thermoset polymer to form a specific shape, thermal energy $\left(25-150^{\circ} \mathrm{C}\right)$ for $>10 \mathrm{~min}$ is required to rapidly crosslink the polymer chains ${ }^{14-16}$. Therefore, it is very difficult to fabricate PDMS in fiber form using a conventional wet spinning method that rapidly extracts fibers through a nozzle. Considering this, a rapid thermal curing process was applied in this study to achieve instant solidification of the thermoset polymer. The PDMS prepolymer and curing agent chemically reacted to form a network structure, thereby rapidly curing the PDMS. During curing, a winder was used to rapidly stretch the PDMS fiber, where the width of the PDMS fiber was controlled by adjusting the tensile force based on the pulling speed. After production, the residual oil was removed to obtain PDMS fibers.

Figure $1 \mathrm{~b}$ shows the chemical crosslinking and solidification mechanisms of PDMS fiber formation using the thermally induced wet-spinning process. The PDMS fiber was formed as the PDMS solution underwent a three-step 


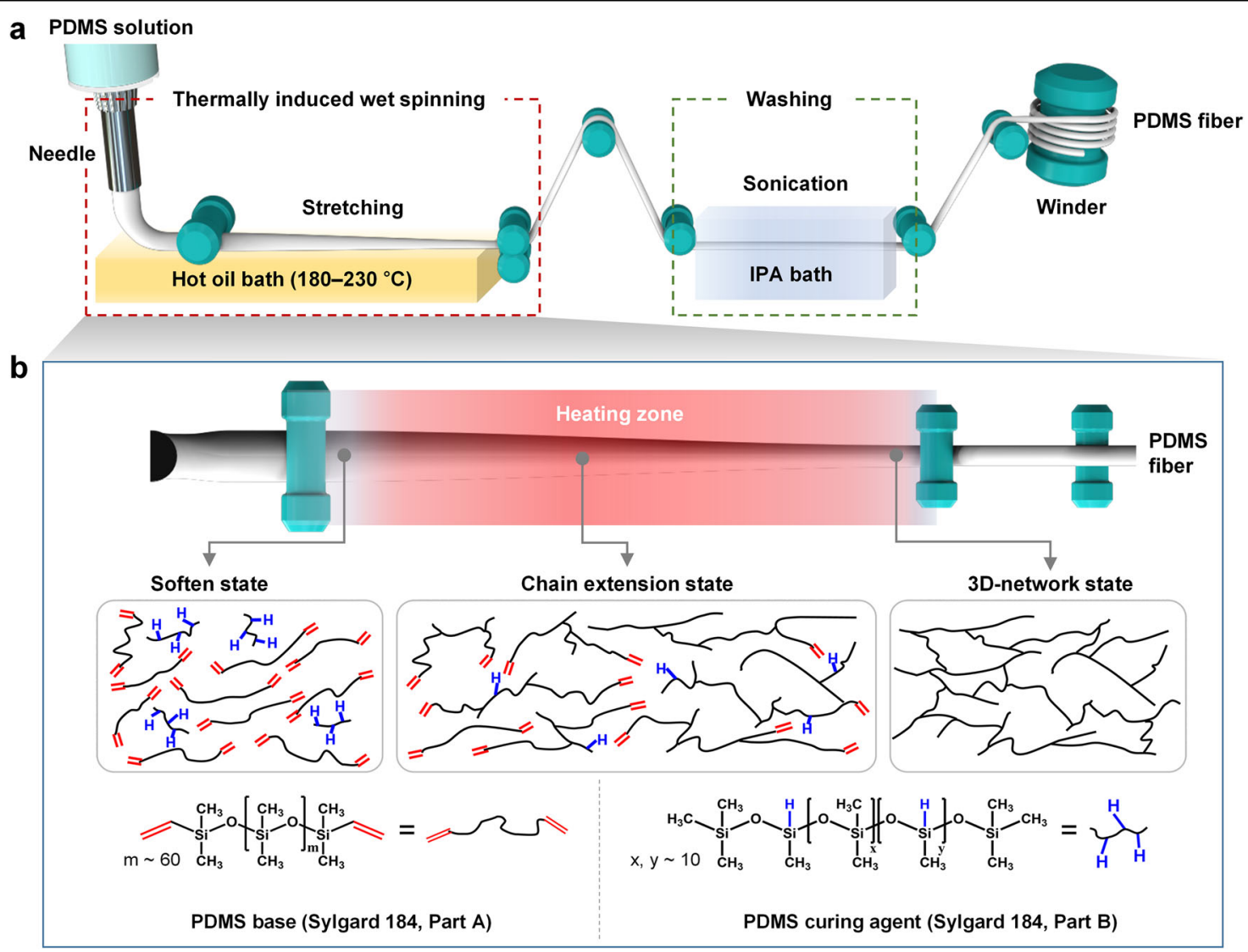

C

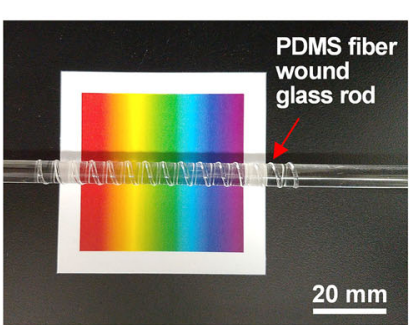

d

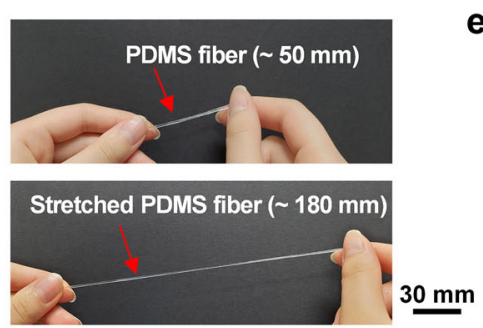

e

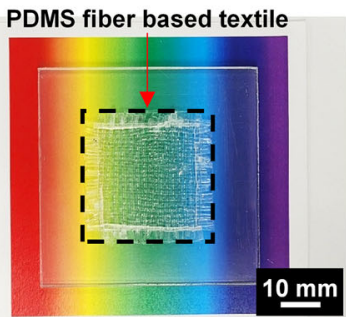

Fig. 1 Stretchable and transparent PDMS fiber. a Schematic illustration of a PDMS fiber fabricated using the thermally induced wet spinning method. $\mathbf{b}$ Mechanism of PDMS solidification on the surface of the hot oil $\left(180-230^{\circ} \mathrm{C}\right)$ to form fibers, followed by stretching using a winder. c Photograph of a PDMS fiber wound around a glass rod. d Photograph of a PDMS fiber stretched from 50 to $180 \mathrm{~mm}$ (260\%). e Photograph of a transparent textile $\left(25 \times 25 \mathrm{~mm}^{2}\right)$ produced by weaving PDMS fibers.

rheology change in high-temperature oil: (1) In the curing process, when the PDMS prepolymer and curing agent were heated by the heat of the oil, their segmental movement and fluidity increased. (2) When the vinyl groups of the PDMS prepolymer and $\mathrm{Si}-\mathrm{H}$ bonds of the curing agent combined, the molecular weight and viscosity increased rapidly as the chain extension and crosslinking reaction proceeded. Because chain extension proceeded before the crosslinking reaction, the polymer chains could temporarily move like a thermoplastic polymer ${ }^{17-19}$. Consequently, the width of the PDMS fiber, which was in a thermoplastic state, could be controlled through a rapid stretching process using a winder. (3) The polymer chains of the PDMS fibers were crosslinked to form three-dimensional networks that did not dissolve in the solvent.

The flexibility and stretchability of the fabricated PDMS fiber were evaluated. The PDMS fiber with a width of up to $1.3 \mathrm{~mm}$ and length of up to $300 \mathrm{~mm}$ could be tightly wound onto a glass rod with a diameter of $6.2 \mathrm{~mm}$ (Fig. 1c). The stretchability of the PDMS fiber with a length of $50 \mathrm{~mm}$ was stably maintained after it was continuously stretched to $180 \mathrm{~mm}$ (260\%) (Fig. 1d). Furthermore, the PDMS fibers exhibited good transparency, and a colorful surface was clearly observed through fabric manufactured using the PDMS fibers $\left(25 \times 25 \mathrm{~mm}^{2}\right)$ (Fig. 1e). 


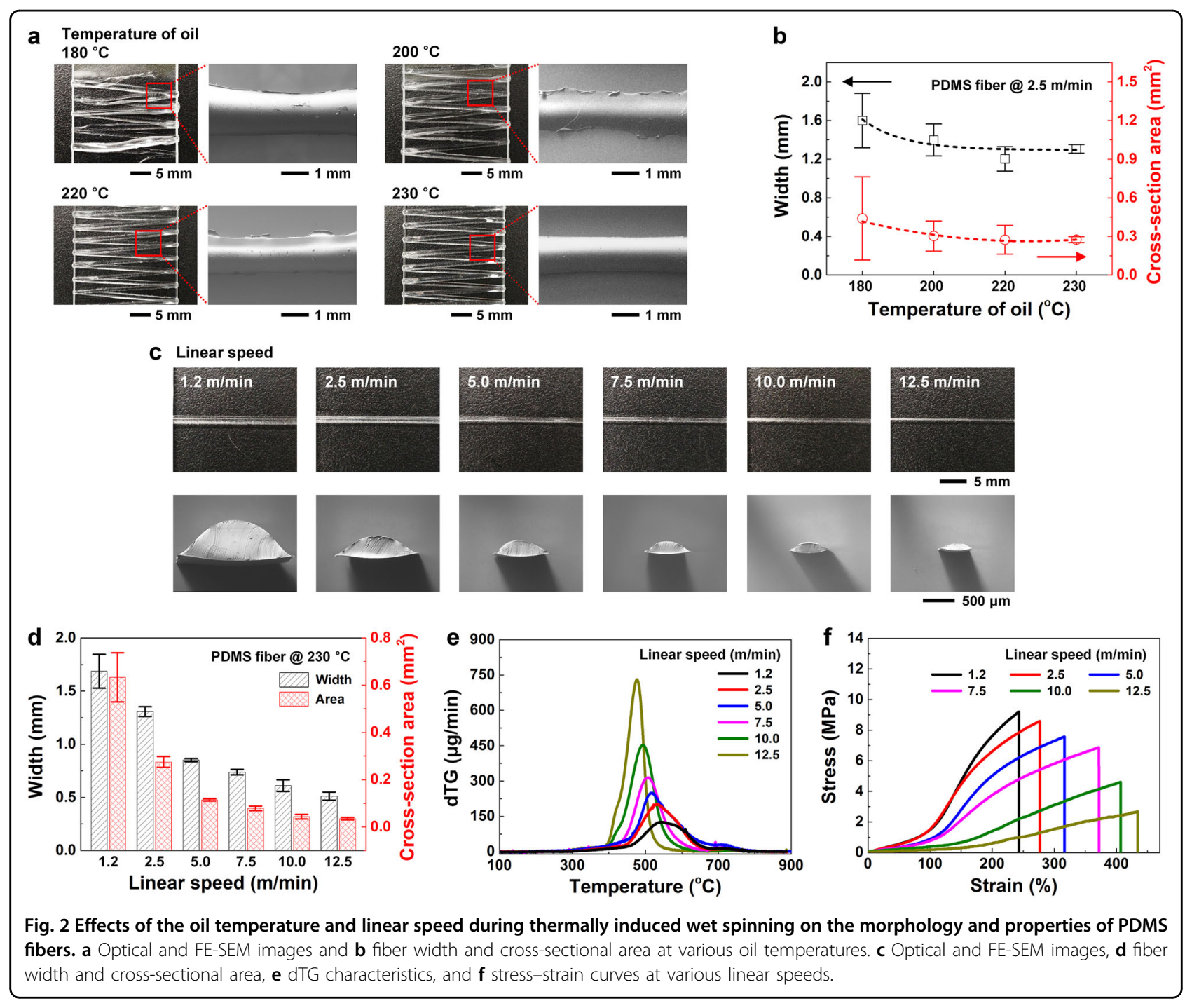

The transparency and mechanical strength of the PDMS fibers were determined by the three-dimensional silicone network formed by the crosslinking of linear silicone molecules. The proposed fabrication method based on rapid thermal curing and stretching was developed to control the shape and properties of the PDMS fibers by improving this three-dimensional silicone network. The curing reaction of a crosslinked polymer, such as PDSM, is a chemical reaction between a liquid prepolymer and a liquid curing agent to produce a solid crosslinked polymer. The curing kinetics of PDMS could be explained by the Arrhenius equation, which represents the correlation between the reaction rate and temperature ${ }^{17,20,21}$ :

$$
\frac{\mathrm{d} p}{\mathrm{~d} t}=k(T) f(\alpha), k(T)=k_{0} e^{-E / R T}
$$

where $\mathrm{d} p / \mathrm{d} t$ is the curing rate, $f(\alpha)$ is a function of the conversion, $k_{0}$ is the pre-exponential constant, $E$ is the activation energy, $R$ is the gas constant, and $T$ is the temperature. According to the Arrhenius equation, the curing rate increases as the curing temperature is increased. Figure 2 shows the changes in the shape and properties of the PDMS fibers according to the oil temperature and linear stretching speed. When the oil temperature was $150-170^{\circ} \mathrm{C}$, the PDMS solution spread like a film on the surface of the oil bath, and the fiber shape could not be maintained. On the other hand, when the oil temperature was $180-230^{\circ} \mathrm{C}$, the PDMS solution maintained its fiber shape on the hot oil surface. The optimum oil temperature was chosen to achieve uniform PDMS fibers; thus, the oil temperature was adjusted between 180 and $230{ }^{\circ} \mathrm{C}$ at a fixed linear speed of $2.5 \mathrm{~m} / \mathrm{min}$. The PDMS curing times decreased to $48 \mathrm{~h}, 35 \mathrm{~min}$, and 10 min with increasing crosslinking temperatures of 25 , 100 , and $150{ }^{\circ} \mathrm{C}$, respectively ${ }^{6,7}$. Thus, exposure of the fluid PDMS solution to a higher oil temperature led to more rapid chain extension and crosslinking to form an elastic and uniform fiber. When the oil temperature was 
low, the PDMS solution was not sufficiently cured and tended to spread in the oil bath to produce a PDMS fiber with a non-uniform thickness. The optical and FE-SEM images of the PDMS fibers (Fig. 2a) revealed that the fiber width was affected by the oil temperature (Fig. 2b), where oil temperatures of $180,200,220$, and $230^{\circ} \mathrm{C}$ led to widths/cross-sectional areas of $1.60 \pm 0.28 / 0.44 \pm 0.32$, $1.40 \pm 0.17 / 0.30 \pm 0.12, \quad 1.20 \pm 0.13 / 0.27 \pm 0.11, \quad$ and $1.31 \pm 0.05 \mathrm{~mm} / 0.28 \pm 0.02 \mathrm{~mm}^{2}$, respectively. Thus, more rapid curing at a higher oil temperature led to a thinner fiber. Furthermore, the standard deviations of the PDMS fiber width and cross-sectional area decreased as the oil temperature was increased, thereby producing a more uniform fiber. Consequently, $230^{\circ} \mathrm{C}$ was chosen as the optimal oil temperature to produce the most uniform PDMS fibers.

The optimal linear speed was chosen to control the width and uniformity of the PDMS fiber. The oil temperature was maintained at $230^{\circ} \mathrm{C}$, and the linear speed was varied $(1.2,2.5,5.0,7.5,10.0$, and $12.5 \mathrm{~m} / \mathrm{min})$. An increase in linear speed led to a larger pulling force on the polymer chains inside the uncured PDMS fiber, thereby forming a finer PDMS fiber. Optical and FE-SEM images were obtained for the PDMS fibers produced at a constant PDMS solution extrusion speed of $60 \mathrm{~mL} / \mathrm{h}$ and linear speeds of 1.2, 2.5, 5.0, 7.5, 10.0, and $12.5 \mathrm{~m} / \mathrm{min}$ (Fig. 2c); the resulting widths/cross-sectional areas were $1.69 \pm$ $0.16 / 0.63 \pm 0.10, \quad 1.31 \pm 0.05 / 0.28 \pm 0.02, \quad 0.85 \pm 0.01 /$ $0.11 \pm 0.005, \quad 0.74 \pm 0.03 / 0.08 \pm 0.01, \quad 0.61 \pm 0.05 / 0.04 \pm$ 0.01 , and $0.51 \pm 0.04 \mathrm{~mm} / 0.03 \pm 0.01 \mathrm{~mm}^{2}$, respectively (Fig. 2d). Thus, a faster linear speed led to a thinner fiber. The standard deviation of the width and cross-sectional area was largest at a linear speed of $1.2 \mathrm{~m} / \mathrm{min}$. Instead, an optimal linear speed of $2.5 \mathrm{~m} / \mathrm{min}$ or higher was chosen to ensure that a uniform PDMS fiber was obtained.

The fiber had a flat shape because the density of PDMS $\left(0.965 \mathrm{~g} / \mathrm{cm}^{3}\right)$ was similar to that of canola oil $(0.92 \mathrm{~g} /$ $\mathrm{cm}^{3}$ ). Thus the PDMS fiber solidified while floating on the hot oil surface. The surface touching the oil surface became spherical owing to surface tension, whereas the surface exposed to air was flat. At a low linear speed, the volume of the spun PDMS fibers was large owing to the effect of gravity: a larger proportion of PDMS sunk below the oil surface and led to a more hemispherical crosssectional fiber shape. Conversely, the PDMS fibers spun at high linear speeds had a reduced volume and were less affected by gravity, thereby resulting in a thinner and flatter cross-section ${ }^{22,23}$.

Derivative thermogravimetric (dTG) curves were plotted to compare the changes in the thermal stability of the PDMS fibers at linear speeds of $1.2-12.5 \mathrm{~m} / \mathrm{min}$ (Fig. 2e). The temperature associated with the greatest weight change due to pyrolysis decreased from 540 to $480^{\circ} \mathrm{C}$ as the linear speed was increased from 1.2 to $12.5 \mathrm{~m} / \mathrm{min}$.
The strain-stress curves of the PDMS fiber revealed that the strain increased and the strength decreased with increasing linear speed owing to less crosslinking (Fig. 2f). The faster the PDMS fiber passed through the hightemperature oil, the shorter the time the PDMS fiber was exposed to a high temperature, which reduced the crosslinking density and three-dimensional silicone network of the PDMS fiber ${ }^{24-26}$. Specifically, the PDMS fibers produced at linear speeds of 1.2, 2.5, 5.0, 7.5, 10.0, and $12.5 \mathrm{~m} / \mathrm{min}$ exhibited strain values of $242,277,316$, 372,407 , and $434 \%$, respectively, and stress values of 9.2, 8.6, 7.6, 6.9, 4.6, and 2.7 MPa, respectively. As the linear speed was increased, the contact time with the hot oil was shortened, and the thermal energy was not sufficiently transferred to the inside of the PDMS fiber. Accordingly, the hydrosilylation reaction, which is a crosslinking reaction, did not proceed sufficiently inside the PDMS fiber. Therefore, as the linear speed was increased, the three-dimensional silicone network formation of the polymer chains of PDMS inside the fiber gradually decreased, resulting in an increase in strain and a decrease in strength. The PDMS fiber fabricated in this study showed higher strain and stress values than previously reported PDMS films and fibers. The strain and stress values of a PDMS cast film fabricated by casting and heating a PDMS prepolymer were $100-170 \%$ and 1.2-7.0 MPa, respectively ${ }^{7,8,27}$. The strain and stress values of a PDMS fiber fabricated by electrospinning were $212 \%$ and $6.0 \mathrm{MPa}$, respectively ${ }^{8}$.

According to thermoset cure kinetics, in the case of the isothermal curing reaction at high temperature, a curing conversion of almost $100 \%$ is reached over time because the polymer chains move freely ${ }^{28-31}$. In contrast, the polymerization and crosslinking reaction in the thermally induced wet spinning method proceeded for a very short time, within $6 \mathrm{~s}$ at a high temperature of $230^{\circ} \mathrm{C}$, followed by rapid cooling. The chain segmental mobility was rapidly reduced with increasing thermal energy; furthermore, the segment mobility was further limited by some of the crosslinked polymer chains in the chain extension process ${ }^{17,31,32}$. Consequently, the curing rate of the PDMS fiber was significantly slowed, and curing conversion was reduced. Therefore, even though the PDMS fiber was left at room temperature $\left(25^{\circ} \mathrm{C}\right)$ for $>48 \mathrm{~h}$, the curing conversion of the PDMS fiber did not change, and the thermal stability and mechanical properties of the PDMS fiber were maintained.

Reversible switching between a transparent and opaque appearance was achieved by stretching and releasing the PDMS fiber after a silica NP layer (thickness: $2 \mu \mathrm{m}$ ) and PDMS capping layer (thickness: $7 \mu \mathrm{m}$ ) were deposited on the PDMS fiber surface (Fig. 3a). Silica NPs were uniformly spray-coated on the surface of the transparent PDMS fiber with a high light transmittance of over $90 \%$, 


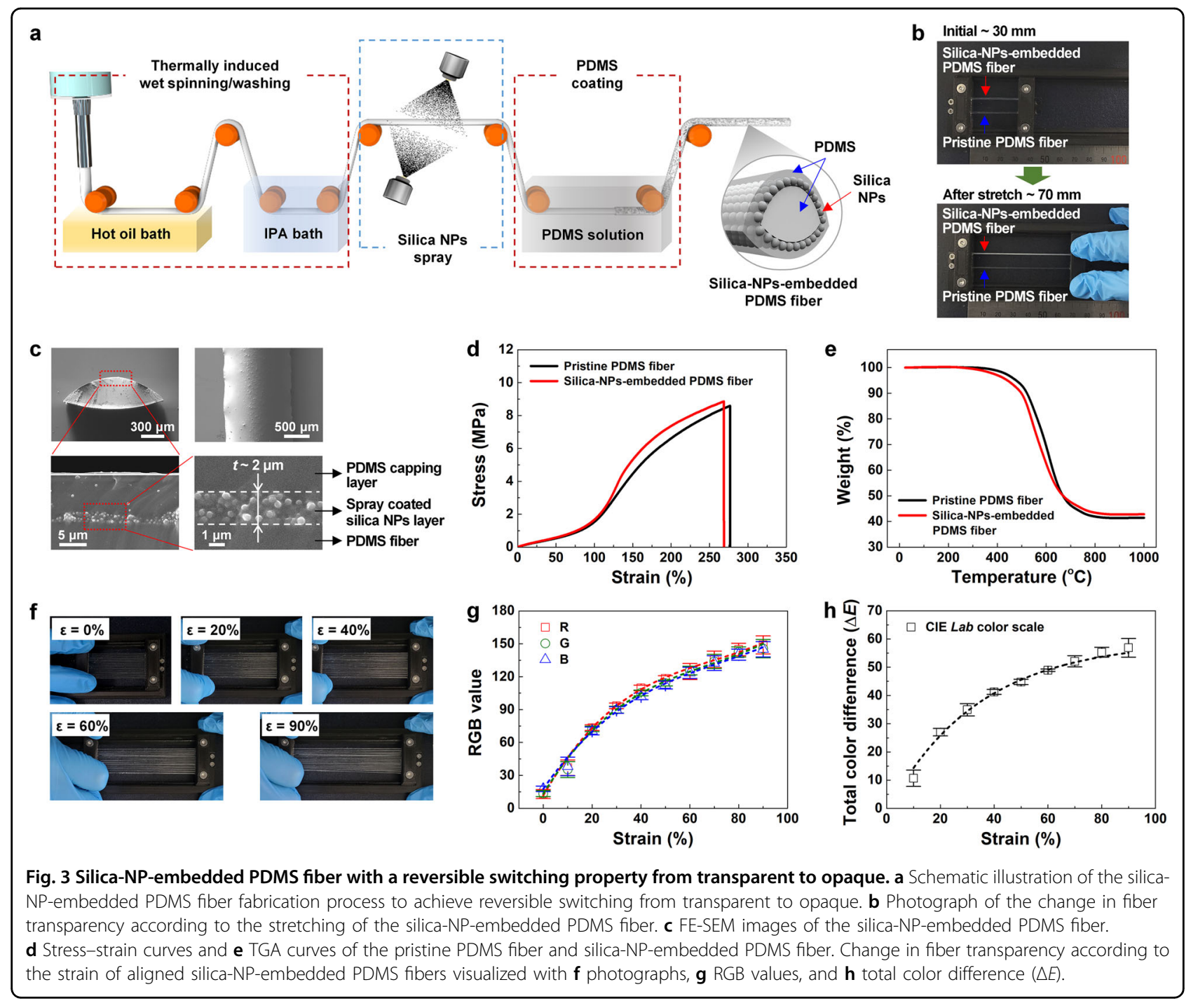

which was subsequently dipped in PDMS solution and thermally cured to obtain a silica-NP-embedded PDMS fiber. The change in the transparency of the silica-NPembedded PDMS fiber (length: $30 \mathrm{~mm}$ ) according to stretching was compared with that of a pristine PDMS fiber (Fig. 3b). Both types of PDMS fibers were visibly transparent in their initial state without any applied force. Spray coating of the transparent PDMS fiber surface with silica NPs led to an opaque appearance owing to the irregular arrangement of the silica NPs. However, the subsequent deposition of the PDMS capping layer restored the transparent appearance because the PDMS solution filled the voids formed between the silica NPs, and they both have similar refractive indices at a wavelength of $632.8 \mathrm{~nm}(\mathrm{PDMS}=1.425$; silica $=1.457)$. The pristine PDMS fiber remained transparent when stretched to $70 \mathrm{~mm}(\sim 130 \%)$, whereas the silica-NP-embedded PDMS fiber became opaque. This was attributed to the separation of the hydrophobic PDMS and hydrophilic silica NPs during stretching, which created voids between the silica NPs and the surrounding PDMS. Optical reflection occurred at the void/silica and void/PDMS interfaces, thereby reducing the transparency. When the fiber was released, the voids between the PDMS and the silica NPs disappeared, and the fiber returned to a transparent state ${ }^{33,34}$. Thus, the appearance of the fiber could be reversibly switched between transparent and opaque via repeated stretching and release of the silicaNP-embedded PDMS fiber.

The surface and cross-section of the silica-NPembedded PDMS fiber were observed using FE-SEM (Fig. 3c), and these observations confirmed that a silica NP layer with a thickness of $\sim 2 \mu \mathrm{m}$ was formed at a depth of $\sim 7 \mu \mathrm{m}$ from the outer surface of the fiber. Considering the PDMS fiber width and thickness of 1.31 and $0.40 \mathrm{~mm}$, respectively, the silica NP layer and added PDMS capping 


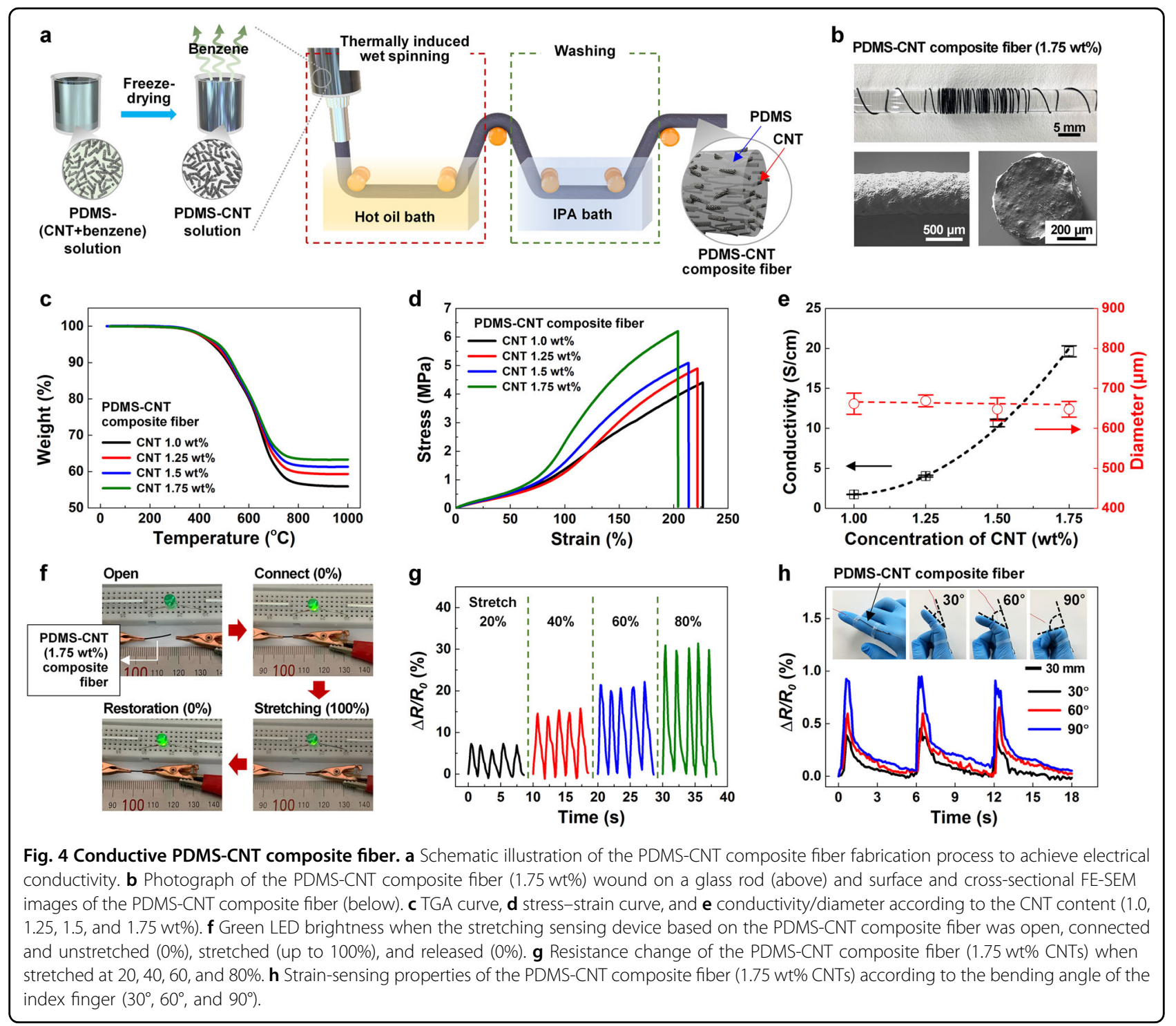

layer were relatively thin. Thus the strain and stress of the pristine PDMS fiber (276\% and $8.6 \mathrm{MPa})$ and silica-NPembedded PDMS fiber (269\% and $8.8 \mathrm{MPa})$ were similar (Fig. 3d). The thermal properties of the silica-NPembedded PDMS fiber were evaluated using TGA (Fig. 3e), where pyrolysis started and ended at 400 and $800^{\circ} \mathrm{C}$ in both fiber types, and the residual amount at $1000{ }^{\circ} \mathrm{C}$ was similar at $\sim 42 \%$. Thus the mechanical and thermal properties of the PDMS fiber were not significantly affected by the embedded silica NPs.

An array of silica-NP-embedded PDMS fibers was prepared by arranging 11 strands side-by-side without gaps, and the samples were placed on a black background to confirm the reversible switching property from transparent to opaque when stretched and released (Fig. 3f). The appearance of the silica-NP-embedded PDMS fibers was assessed when they were stretched to $20 \%, 40 \%, 60 \%$, and
$90 \%$. The transparent fibers gradually turned white as the stretching length was increased. The change in transparency was quantified based on the RGB and CIE $L^{*} a^{*} b^{*}$ color space measurements (Fig. $3 g$, h). The fiber in the unstretched initial state appeared black owing to the black background (13 (R), 13.4 (G), and 17.8 (B)), and it turned white when stretched to 90\% (149 (R), $145.6(\mathrm{G}), 450$ (B)). The total color difference $(\Delta E)$ value was obtained by extracting CIE $L^{*} a^{*} b^{*}$, which is a three-dimensional numerical value of color, and a quasilinear tendency similar to that of the RGB values was observed.

Conductive PDMS-CNT composite fibers were prepared by adding CNTs to the PDMS and were used as a strain sensor (Fig. 4a). The PDMS-CNT composite fiber was wound around a $6.2-\mathrm{mm}$ diameter glass rod and observed using FE-SEM (Fig. 4b). The PDMS-CNT composite fiber was successfully solidified when 
immersed in high-temperature oil. Furthermore, the CNTs were denser $\left(1.6 \mathrm{~g} / \mathrm{cm}^{3}\right)$ than PDMS $(0.965 \mathrm{~g} /$ $\mathrm{cm}^{3}$ ), thereby resulting in a composite with a higher density than that of canola oil $\left(0.92 \mathrm{~g} / \mathrm{cm}^{3}\right)$. Thus, hydraulic pressure was applied uniformly to the entire fiber as it sunk below the oil surface, and fiber with a circular cross-section was formed (Supplementary Fig. S1).

The thermal properties of the PDMS-CNT composite fiber were measured using TGA from 0 to $1000^{\circ} \mathrm{C}$ (Fig. 4c). PDMS-CNT composite fiber decomposition started at $\sim 400^{\circ} \mathrm{C}$, after which the sample weight plateaued starting at $800^{\circ} \mathrm{C}$. The PDMS-CNT composite fibers were not completely decomposed, even at temperatures above $800^{\circ} \mathrm{C}$. The PDMS-CNT composite fibers with CNT contents of 1.0, 1.25, 1.5, and $1.75 \mathrm{wt} \%$ exhibited a positive correlation with the residual amounts of CNTs at $1000{ }^{\circ} \mathrm{C}$, namely, 55.9, 59.3, 61.3, and 63.3\%.

An analysis of the strain-stress properties of the PDMSCNT composite fibers revealed that an increase in the CNT content of the PDMS-CNT composite fibers between $1.0,1.25,1.5$, and $1.75 \mathrm{wt} \%$ led to a gradual decrease in strain value $(227,222,214$, and $204 \%$, respectively) and a gradual increase in stress $(4.4,4.9,5.1$, and $6.2 \mathrm{MPa}$, respectively) (Fig. 4d). Composite fibers comprising a flexible polymer mixed with a powder/particle tended to exhibit a decrease in flexibility as the amount of added powder/particle was increased owing to interference with the polymer chain interactions ${ }^{35,36}$. The stress applied to the fibers was effectively dispersed in the CNTs owing to their excellent mechanical properties and strong adhesion at the $\mathrm{CNT} /$ polymer matrix interface.

The conductivity and diameter of the PDMS-CNT composite fibers were evaluated according to the CNT content (Fig. 4e). The conductivity increased with increasing CNT content, where the conductivity with 1.0 , $1.25,1.5$, and $1.75 \mathrm{wt} \% \mathrm{CNTs}$ was $1.7 \pm 0.03,4.0 \pm 0.11$, $10.7 \pm 0.48$, and $19.6 \pm 0.69 \mathrm{~S} / \mathrm{cm}$, respectively. Despite the increase in the CNT content, the respective fiber diameters remained relatively constant at $662 \pm 26,669 \pm 15$, $648 \pm 29$, and $647 \pm 20 \mu \mathrm{m}$ for $1.0,1.25,1.5$, and $1.75 \mathrm{wt} \%$ CNTs. The conductivity of an object is considered in relation to the specific resistance of the conductive material contained in the object, the junction resistance in the conduction path at the contact point between conductive materials, and the resistance due to electron tunneling between noncontact materials. The electron tunneling resistance played the most decisive role in the overall resistance of a composite comprising insulating PDMS and conductive CNTs, as the polymer formed a thin layer between the CNTs, preventing their direct connection. An increase in the CNT content of the fiber reduced the minimum distance between the $\mathrm{CNTs}$ to form an electrical-percolation network, thereby increasing the conductivity ${ }^{37}$.
The 1.75-wt\% PDMS-CNT composite fiber offered the highest electrical conductivity and was applied in a stretch sensing device (Fig. 4f). Specifically, the PDMS-CNT composite fiber was used as wiring (length: $5 \mathrm{~mm}$ ) for an electrode to apply voltage to a green light-emitting diode (LED). The PDMS-CNT composite fiber was stretched by $100 \%$ to $10 \mathrm{~mm}$ at an applied voltage of $5 \mathrm{~V}$, which led to an increase in the resistance and a less bright LED. After releasing the stretched fiber back to $5 \mathrm{~mm}$, the resistance decreased, and the brightness of the LED was restored. Fig $4 \mathrm{~g}$ shows the change in the $\Delta R / R_{0}$ value according to the stretching of the PDMS-CNT composite fiber, where the fiber stretched at $20,40,60$, and $80 \%$ exhibited a linear increase in the $\Delta R / R_{0}$ value: $6.8,14.9,21.0$, and $30.4 \%$, respectively. This behavior remained consistent after five repeated stretch and release cycles.

The PDMS-CNT composite fiber was attached to a human index finger to detect finger bending based on fiber stretching (Fig. 4h). Thus, the real-time change in the resistance of the PDMS-CNT composite fiber was gradually increased by $0.4,0.6$, and $0.9 \%$ when the finger was bent at $30^{\circ}, 60^{\circ}$, and $90^{\circ}$, respectively, for a total of three times. The response of the sensor and return characteristics were evaluated based on the response/ relaxation time, which was maintained at a constant value of $\sim 0.5 / 1.0 \mathrm{~s}$, even when the bending angle was changed.

Figure 5 shows the fabrication, pore distribution, and thermal insulation properties of the porous PDMS fiber. Pores were formed inside the PDMS fiber by embedding PMMA particles within the PDMS during fiber formation and selectively dissolving and removing the PMMA once the PDMS fiber was formed to leave a porous skeleton (Fig. 5a). The porous PDMS fiber had an opaque white appearance owing to the internal pores and exhibited sufficient flexibility to be wound around a $6.2-\mathrm{mm}$-diameter glass rod (inset in Fig. 5a).

The FE-SEM images of the porous PDMS fiber produced using PDMS solutions with PMMA powder proportions of $10,20,30,40$, and $50 \mathrm{wt} \%$ were compared (Fig. 5b). As the PMMA content was increased to $40 \mathrm{wt} \%$, the width and thickness of the PDMS fiber increased, as did the number of internal and surface pores (Supplementary Fig. S2). PMMA is a thermoplastic polymer with a $T_{\mathrm{g}}$ of $85-165^{\circ} \mathrm{C}$; thus, the mobility and flexibility of the polymer chains increased when exposed to hightemperature oil $\left(230^{\circ} \mathrm{C}\right)^{38}$. This resulted in an increase in the volume of PMMA. The PDMS solidified while the PMMA was in the increased volume state; thus, the width and thickness of the PMMA particle-embedded PDMS fiber increased with increasing PMMA content. Furthermore, the porosity of the PMMA particle-embedded PDMS fibers increased with increasing PMMA content. However, those formed at $50 \mathrm{wt} \%$ PMMA were not capable of maintaining the pore structure and subsequently 


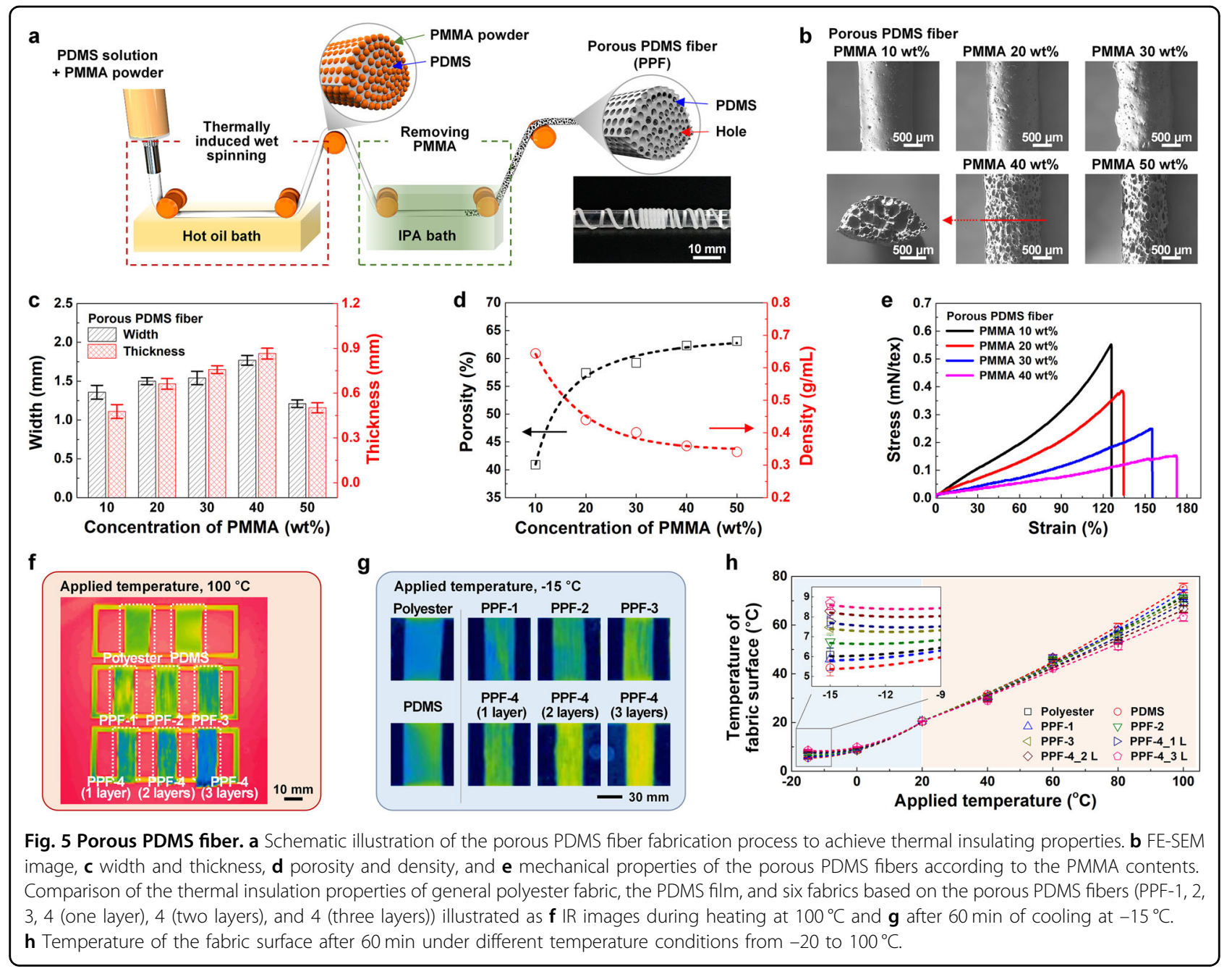

contracted. Consequently, the widths/thicknesses of the porous PDMS fibers were $1.36 \pm 0.09 / 0.48 \pm 0.05,1.50 \pm$ $0.04 / 0.66 \pm 0.04,1.54 \pm 0.09 / 0.76 \pm 0.03 \mathrm{~mm}$, and $1.77 \pm$ $0.06 / 0.86 \pm 0.04$ at $10,20,30$, and $40 \mathrm{wt} \%$ PMMA, respectively, and they decreased to $1.21 \pm 0.05 / 0.50 \pm$ $0.03 \mathrm{~mm}$ at $50 \mathrm{wt} \%$ PMMA (Fig. 5c). Furthermore, the porosity of the porous PDMS fibers with 10, 20, 30, and 40 wt\% PMMA increased to 40.9, 57.4, 59.2, and 62.3\%, respectively, with increasing PMMA content, while the density gradually decreased to $0.64,0.44,0.40$, and $0.36 \mathrm{~g} /$ $\mathrm{mL}$, respectively (Fig. $5 \mathrm{~d}$ ). In the case of the porous PDMS fiber with $10 \mathrm{wt} \%$ PMMA, PMMA existed in the form of isolated grains rather than forming connected grains because the amount of PMMA powder mixed into the PDMS matrix was small. Therefore, during the PMMA removal process, PMMA existing in the form of isolated grains inside the PDMS fiber could not be removed, and the porosity of the PDMS fiber with $10 \mathrm{wt} \%$ PMMA decreased. However, the porous PDMS fiber with $50 \mathrm{wt} \%$ PMMA underwent pore size contraction during drying, and the porosity and density were similar to those of the porous PDMS fiber with $40 \mathrm{wt} \%$ PMMA. Overall, these results demonstrated that the pore structure of the PDMS fiber was well formed up to a PMMA content of $40 \mathrm{wt} \%$. The mechanical properties of the porous PDMS fiber were evaluated based on the stress-strain curve, where the strain gradually increased and the stress decreased between 10 and $40 \mathrm{wt} \%$. Specifically, the PDMS fibers with $10,20,30$, and $40 \mathrm{wt} \%$ PMMA exhibited strain values of $126,134,155$, and $172 \%$, respectively, and stress values of $0.55,0.38,0.25$, and $0.15 \mathrm{mN} /$ tex, respectively (Fig. 5e). Consequently, all of the porous PDMS fibers exhibited excellent elongation of $100 \%$ or more.

The thermal insulation properties of a general polyester fabric $(t \sim 0.7 \mathrm{~mm})$, PDMS film $(t \sim 0.4 \mathrm{~mm})$, and four porous PDMS fiber fabrics (PPF-1, 2, 3, and 4) $(t \sim$ $0.7 \mathrm{~mm}$ ) were compared while heating at temperatures up to $100^{\circ} \mathrm{C}$ and cooling to $-15^{\circ} \mathrm{C}$ (Fig. $5 \mathrm{f}$, g). The samples were placed on heating or cooling substrates, and the temperature was saturated for $20 \mathrm{~min}$ (Supplementary 
Figs. S3 and S4). The IR images clearly illustrated that the porous PDMS fiber prepared with 40\% PMMA (PPF-4) exhibited the best thermal insulation effect, which was attributed to its high porosity. The thermal conductivities of the pure PDMS film, PPF-1, PPF-2, PPF-3, and PPF- 4 were $0.1606 \pm 0.0004,0.1575 \pm 0.0032,0.1385 \pm 0.0010$, $0.0930 \pm 0.0001$, and $0.0790 \pm 0.0009 \mathrm{~W} \cdot \mathrm{m}^{-1} \cdot \mathrm{K}^{-1}$, respectively (Supplementary Fig. S5) The thermal conductivity decreased as the number of pores inside the PDMS fiber increased. In addition, the thermal insulation effect improved as the number of porous PDMS fiber layers in the fabric was increased from one to three. When exposed to heating conditions of $100^{\circ} \mathrm{C}$, the temperatures of the polyester fabric, PDMS thin film, and PPF-1, 2, 3, 4 (one layer), 4 (two layers), and 4 (three layers) were $71.0 \pm 0.4$, $75.3 \pm 1.9, \quad 72.5 \pm 2.0, \quad 71.0 \pm 1.4, \quad 69.4 \pm 1.7, \quad 68.9 \pm 0.9$, $66.7 \pm 1.4$, and $63.2 \pm 1.6^{\circ} \mathrm{C}$, respectively, while the corresponding values under cooling conditions of $-15^{\circ} \mathrm{C}$ were $6.1 \pm 0.3,5.4 \pm 0.4,5.9 \pm 0.6,6.8 \pm 0.3,7.5 \pm 0.3,7.8 \pm$ $0.3,8.3 \pm 0.4$, and $8.7 \pm 0.3{ }^{\circ} \mathrm{C}$, respectively. In comparison with the polyester fabric and PDMS thin film, the maximum temperature difference of the PPF-4 (three layers) fabric was up to $\sim 10^{\circ} \mathrm{C}$ at $100^{\circ} \mathrm{C}$ and up to $\sim 3^{\circ} \mathrm{C}$ at $-15^{\circ} \mathrm{C}$ (Fig. 5h).

\section{Conclusion}

A thermally induced wet spinning method was successfully used to fabricate pristine PDMS fibers, silica-NPembedded PDMS fibers, PDMS-CNT composite fibers, and porous PDMS fibers. The pristine PDMS fibers were transparent and highly elastic and were produced by wet spinning uncured PDMS comprising a mixture of prepolymer and crosslinking agent into a coagulation bath containing oil with no solvent at a controlled temperature of $180-230^{\circ} \mathrm{C}$. The width and corresponding mechanical properties of the fiber were adjustable. The wet-spun polymer chain underwent rapid chemical crosslinking owing to heat transfer from the high-temperature oil and stretching at a speed of $1.2-12.5 \mathrm{~m} / \mathrm{min}$. This thermally induced wet spinning method was ecofriendly and economical, as edible canola oil was used for coagulation, and it could be performed using conventional wet spinning equipment. Three functional fibers were fabricated by modifying the pristine PDMS fiber. A PDMS fiber embedded with silica NPs was fabricated to achieve switchable optical transparency/opacity properties according to the tension and contraction of the fiber. This reversible switching could be utilized for smart blinds, security, heat/solar energy gain control, and color strain sensors. A conductive PDMS fiber was fabricated by mixing conductive CNTs with uncured PDMS. This material showed promising applicability in wearable devices, such as strain sensors to detect body movements. A porous PDMS fiber with a porosity of up to $62 \%$ was fabricated to achieve thermal insulation. This fiber could be used as an energy-saving material for wearable devices. The proposed PDMS fiber fabricated by the thermally induced wet spinning method had a three-dimensional crosslinked network. Therefore, thermal and chemical stability could be secured even when it was exposed to the external environment for a long period of time. In addition, optical, electrical, and thermal insulating properties could be conferred onto these PDMS fibers, thereby providing the possibility of stably fabricating PDMS fibers mixed with various functional materials.

\section{Acknowledgements}

This work was supported by the National Research Foundation of Korea (NRF) grants funded by the Korean government (MSIT) (2018M3A7B4070987, 2019R1A2C2010614, 2019R1C1C1010688, 2020R1A5A1019131, and 2021R111A1A01060148).

\section{Author contributions}

T.L., S.M.J., and S.J. conceived and designed the study. J.H.L., T.L., K.S., J.P., and J. Y. carried out the experiments. J.H.L., T.L., and S.-M.J. analyzed the data. T.L., S.M.J., and S.J. wrote the paper. All authors contributed to discussions regarding the research.

\section{Competing interests}

The authors declare no competing interests.

\section{Publisher's note}

Springer Nature remains neutral with regard to jurisdictional claims in published maps and institutional affiliations.

Supplementary information The online version contains supplementary material available at https://doi.org/10.1038/s41427-022-00359-7.

Received: 21 October 2021 Revised: 15 December 2021 Accepted: 21 December 2021.

Published online: 25 February 2022

\section{References}

1. Kim, H.J., Sim, K., Thukral, A. \& Yu, C. Rubbery electronics and sensors from intrinsically stretchable elastomeric composites of semiconductors and conductors. Sci. Adv. 3, e1701114 (2017).

2. Jeong, S. H., Zhang, S., Hjort, K., Hilborn, J. \& Wu, Z. PDMS-based elastomer tuned soft, stretchable, and sticky for epidermal electronics. Adv. Mater. 28, 5830-5836 (2016)

3. Gao, Y. et al. Wearable microfluidic diaphragm pressure sensor for health and tactile touch monitoring. Adv. Mater. 29, 1701985 (2017).

4. Yun, T. G. et al. All-transparent stretchable electrochromic supercapacitor wearable patch device. ACS Nano 13, 3141-3150 (2019).

5. Yoo, D., Johnson, T. W., Cherukulappurath, S., Norris, D. J. \& Oh, S.-H. Templatestripped tunable plasmonic devices on stretchable and rollable substrates. ACS Nano 9, 10647-10654 (2015).

6. Hopf, R. et al. Experimental and theoretical analyses of the age-dependent large-strain behavior of Sylgard 184 (10:1) silicone elastomer. J. Mech. Behav. Biomed. Mater. 60, 425-437 (2016).

7. Johnston, I. D., McCluskey, D. K., Tan, C. K. L. \& Tracey, M. C. Mechanical characterization of bulk Sylgard 184 for microfluidics and microengineering. J. Micromech. Microeng. 24, 035017 (2014).

8. Niu, H., Wang, H., Zhou, H. \& Lin, T. Ultrafine PDMS fibers: preparation from in situ curing-electrospinning and mechanical characterization. RSC Adv. 4, 11782-11787 (2014)

9. Wang, D., Sheng, B., Peng, L., Huang, Y. \& Ni, Z. Flexible and optical fiber sensors composited by graphene and PDMS for motion detection. Polymers 11, 1433 (2019). 
10. Guo, J. et al. Stretchable and highly sensitive optical strain sensors for humanactivity monitoring and healthcare. ACS Appl. Mater. Interfaces 11, 33589-33598 (2019).

11. Lu, S., Duan, X., Han, Y. \& Huang, H. Silicone rubber/polyvinylpyrrolidone microfibers produced by coaxial electrospinning. J. Appl. Polym. Sci. 128, 2273-2276 (2013).

12. Agarwal, A., Raheja, A., Natarajan, T. S. \& Chandra, T. S. Development of universal $\mathrm{pH}$ sensing electrospun nanofibers. Sens. Actuators B Chem. 161, 1097-1101 (2012).

13. Tassanawat, S., Phandee, A., Magaraphan, R., Nithitanakul, M. \& Manuspiya, H. $\mathrm{pH}$-sensitive PP/clay nanocomposites for beverage smart packaging. 2007 2nd IEEE International Conference on Nano/Micro Engineered and Molecular Systems, 478-482 (2007).

14. Kim, S.-S. et al. Degradable thermoset fibers from carbohydrate-derived diols via thiol-ene photopolymerization. ACS Appl. Polym. Mater. 1, 2933-2942 (2019).

15. Shanmuganathan, K., Sankhagowit, R. K., Iyer, P. \& Ellison, C. J. Thiol-ene chemistry: a greener approach to making chemically and thermally stable fibers. Chem. Mater. 23, 4726-4732 (2011).

16. Shanmuganathan, K., Elliot, S. M., Lane, A. P. \& Ellison, C. J. Highly stretchable thermoset fibers and nonwovens using thiol-ene photopolymerization. ACS Appl. Mater. Interfaces 6, 14259-14265 (2014).

17. Hong, I.-K. \& Lee, S. Cure kinetics and modeling the reaction of silicone rubber. J. Ind. Eng. Chem. 19, 42-47 (2013).

18. Tao, Q., Pinter, G., Antretter, T., Krivec, T. \& Fuchs, P. Model free kinetics coupled with finite element method for curing simulation of thermosetting epoxy resins. J. Appl. Polym. Sci. 135, 46408 (2018).

19. Rabearison, N., Jochum, C. \& Grandidier, J. C. A cure kinetics, diffusion controlled and temperature dependent, identification of the Araldite LY556 epoxy. J. Mater. Sci. 46, 787-796 (2011).

20. Sbirrazzuoli, N., Brunel, D. \& Elegant, L. Different kinetic equations analysis. J. Therm. Anal. 38, 1509-1524 (1992).

21. Stieghorst, J. \& Doll, T. Rheological behavior of PDMS silicone rubber for 3D printing of medical implants. Addit. Manuf. 24, 217-223 (2018).

22. Jones, S. G., Abbasi, N., Ahuja, A., Truong, V. \& Tsai, S. S. H. Floating and sinking of self-assembled spheres on liquid-liquid interfaces: rafts versus stacks. Phys. Fluids 27, 072102 (2015).

23. Hui, C.-Y. \& Jagota, A. Planar equilibrium shapes of a liquid drop on a membrane. Soft Matter 11, 8960-8967 (2015).
24. Winter, H. H. \& Chambon, F. Analysis of linear viscoelasticity of a crosslinking polymer at the gel point. J. Rheol. 30, 367-382 (1986).

25. Katashima, T. Rheological studies on polymer networks with static and dynamic crosslinks. Polym. J. 53, 1073-1082 (2021).

26. Calvet, D., Wong, J. Y. \& Giasson, S. Rheological monitoring of polyacrylamide gelation: importance of cross-link density and temperature. Macromolecules 37, 7762-7771 (2004).

27. Tang, Z., Gao, Z., Jia, S., Wang, F. \& Wang, Y. Graphene-based polymer bilayers with superior light-driven properties for remote construction of 3D structures. Adv. Sci. 4, 1600437 (2017).

28. Feve, M. Network formation in an epoxy system: a kinetics, viscometric and viscoelastic study. Makromol. Chem. Macromol. Symposia 30, 95-107 (1989).

29. Enns, J. B. \& Gillham, J. K. Time-temperature-transformation (TTT) cure diagram: Modeling the cure behavior of thermosets. J. Appl. Polym. Sci. 28 2567-2591 (1983).

30. Roller, M. B. Rheology of curing thermosets: a review. Polym. Eng. Sci. 26, 432-440 (1986).

31. Zhao, L. \& Hu, X. Autocatalytic curing kinetics of thermosetting polymers: a new model based on temperature dependent reaction orders. Polymer 51, 3814-3820 (2010).

32. Konuray, O. et al. Time-temperature-transformation (TTT) diagram of dualcurable epoxy thermosets obtained via two sequential epoxy-amine condensations. Thermochim. Acta 678, 178305 (2019).

33. Ge, D. et al. A robust smart window: reversibly switching from high transparency to angle-independent structural color display. Adv. Mater. 27, 2489-2495 (2015)

34. Kim, H.-N., Ge, D., Lee, E. \& Yang, S. Multistate and on-demand smart windows. Adv. Mater. 30, 1803847 (2018)

35. Bigg, D. M. Mechanical properties of particulate filled polymers. Polym. Compos. 8, 115-122 (1987).

36. Wang, $X$. et al. Mechanical properties of polymer composites reinforced by functionalized graphene prepared via direct exfoliation of graphite flakes in styrene. RSC Adv. 6, 112486-112492 (2016).

37. Liu, H. et al. Electrically conductive polymer composites for smart flexible strain sensors: a critical review. J. Mater. Chem. C. 6, 12121-12141 (2018).

38. Gurina, D., Budkov, Y. \& Kiselev, M. Molecular dynamics study of the swelling of poly(methyl methacrylate) in supercritical carbon dioxide. Materials 12, 3315 (2019). 